TOM HOLERT

\title{
„MY PHONE'S ON VIBRATE FOR YOU \\ ÜBER INNERVATION UND VIBROTAKTILE \\ KOMMUNIKATION NACH WALTER BENJAMIN
}

\begin{abstract}
Und sobald unserer Anruf erklungen ist, - mitten in der bilderreichen Nacht, die sich nur unseren Ohren auftut, ein Geräusch leise - abstrakt - das Geräusch der unterdrückten Entfernung - und die Stimme des lieben Wesens kommt zu uns.

Marcel Proust, in der Übersetzung von Walter Benjamin und Franz Hessel ${ }^{1}$
\end{abstract}

\section{Zuckungen eines tönenden Stummels}

„Vibrate“, 2003 veröffentlicht auf der CD Want One (Dreamworks/Universal) des kanadischen Sängers und Komponisten Rufus Wainwright, ist eine mit klagender Stimme vorgetragene Ballade, die das Proust'sche Drama der telekommunikativ gestützten Erwartung paraphrasiert, wie es der junge Marcel in Doncières erlebt, als er dem Anruf seiner Großmutter aus Paris entgegenfiebert. Wainwright erzählt singend von einem Mann, der durch die Nacht zieht und dabei ein Mobiltelefon bei sich trägt, das auf den Vibrationsmodus eingestellt ist. Auch hier kann dem Drama der Antizipation der plötzlichen Aufhebung der Distanz durch die Ankunft der Stimme des geliebten oder auch begehrten Wesens beigewohnt werden. Wainwright ergänzt die akustische durch eine haptische

1 Proust, Marcel: „Guermantes“, in: Benjamin, Walter: Gesammelte Schriften, hrsg. v. Rolf Tiedemann/Hermann Schweppenhäuser, Supplement III, hrsg. v. Hella Tiedemann-Bartels, Frankfurt a.M. 1987, S. 128. Den Hinweis auf Proust verdanke ich Volker Roloff und Peter Gendolla. Zu Dank bin ich auch und vor allem dem SFB/FK „Medienumbrüche“ verpflichtet, dessen Einladung zu einem Aufenthalt als Gastwissenschaftler im November 2005 die Entstehung dieses Textes ermöglicht hat. 
Komponente, doch freilich hat auch Proust diese Dimension schon berücksichtigt. Denn der erste Versuch seiner Großmutter, ihn telefonisch zu erreichen, schlug fehl: Als Marcel die Telefonzelle betrat, ,sprach jemand, der offenbar nicht wußte, daß niemand da war, ihm zu antworten, denn als ich den Hörer an mich nahm, fing dieses Stück Holz zu reden an wie Hanswurst ${ }^{\star 2}$. Eindringlich schildert Proust seine Verzweiflung, das hölzerne Ding zur Ruhe zu bringen, „die Zuckungen dieses tönenden Stummels“ zu beenden, um wieder Zugang zur Persönlichkeit und zur Stimme des geliebten telefonischen Gegenübers zu erhalten.

Die poetische Aufmerksamkeit, die Proust und Wainwright den Aufmerksamkeitstechnologien in den Alltagen des frühen 20. und des frühen 21. Jahrhunderts schenken, soll den Ausgangspunkt für eine Betrachtung der heutigen „Spannungen“ bilden, die nach Walter Benjamin, dem Übersetzer Prousts, ,die Technisierung mit ihren Folgen in den großen Massen erzeugt hat ${ }^{* 3}$ und welche nunmehr - in einem von Benjamin, wie zu zeigen sein wird, bereits vorweggenommenen Maß - individualisiert und ,personalisiert' sind.

Mit oft doppelbödigen, von innuendo und double entendre, den Codes des andeutend-anspielenden Sprechens durchwirkten Liedern feiert der Singer/Songwriter Wainwright seit seinem Debütalbum von 1998 immer größere Erfolge. Mitte des ersten Jahrzehnts des neuen Jahrhunderts gilt er als einer der Lieblingsentertainer eines nicht zuletzt queeren, homo- oder metrosexuellen metropolitanen Publikums in Nordamerika und Europa. Seine bittersüß getexteten Stücke erzählen von seltsamen alläglichen Begebenheiten, komplizierten Familienverhältnissen und nostalgischen Obsessionen für das 19. Jahrhundert. In Projektionen jenes „,blissful, wonderful kind of gay lifestyle ${ }^{64}$, den er schmachtend und ironisch propagiert, huldigt er Schubert und Verdi, erkundet Kalifornien und Kathedralen, schwärmt vom Berlin der Weimarer Republik oder einem ähnlich geschichtsentrückten Manhattan. Dazu bevorzugt Wainwright, der eine voluminöse und zugleich unkonventionell-charakteristische Stimme als Markenzeichen pflegt, das opulente, bisweilen opernhafte Arrangement. Obwohl er sich in „Vibrate“ über weite Strecken mit einem für seine Verhältnisse sparsamen Streichersatz begnügt, steigert

2 Ebd., S. 129.

3 Benjamin, Walter: „Das Kunstwerk im Zeitalter seiner technischen Reproduzierbarkeit“ (2. Fassung), in: Gesammelte Schriften, hrsg. v. Rolf Tiedemann/Hermann Schweppenhäuser, Bd. 7, Frankfurt a.M. 1989, S. 377.

4 Zit. n. Udovitch, Mim: „The Importance of Being Rufus“, in: Rolling Stone, 10.06.1999, S. 57-62, hier S. 60. 
Wainwright auch in diesem Song zum Ende hin die Üppigkeitswerte und krönt ihn durch die Stimmen eines dreißigköpfigen Knabenchors.

All dies sei erwähnt, um zu zeigen, dass sich Technologiediskurse höchst selten in die musikalische Textur von Wainwrights Kompositionen (etwa durch eine ,technoide', ,elektronische“ Anmutung) oder in die Motivik seine Liedtexte verirren. Die reichlich verteilten Camp-Referenzen und romantischen Hyperbeln rufen eher das kulturell und autobiographisch Vergangene und (durchaus im Benjaminschen Sinn) Veraltete auf und an, während die technischen Umwelten und Telekommunikationen der Gegenwart aus diesem poetischen Universum sonst wie verbannt scheinen. Der Song „Vibrate“ stellt damit im Kontext des Schaffens dieses Popkünstlers eine Ausnahme dar:

\author{
My phone's on vibrate for you \\ Electroclash is karaoke too \\ I tried to dance Britney Spears \\ I guess I'm getting on in years \\ My phone's on vibrate for you \\ God knows what all these new drugs do \\ I guess to have no more fears \\ But still I always end up in tears \\ My phone's on vibrate for you \\ But still I never ever feel from you \\ Pinocchio's now a boy who wants to turn back into a toy \\ So call me \\ Call me the morning, call me in the night \\ So call me \\ Call me anytime you like \\ My phone's on vibrate \\ For you, for you
}

„Vibrate“ versammelt und verknüpft Motive auf eine Weise, dass sich ein spezifisches Verhältnis von Medienevolution, Medienhandeln, Medienwissen und Subjektivierung abzeichnet. Wainwright entwirft hier Bilder und Stimmungen einer Beziehung zwischen Mensch und Technik, die das Unausweichliche der Medientechnologie, die Alltäglichkeit des Aufenthalts in kybernetisch-telekommunikativen Zonen ebenso umspielen wie sie Möglichkeiten skizzieren, die Informationalisierung und Technifizierung von Affekten und Emotionen ästhetisch zu bearbeiten.

Wainwright hat wissen lassen, dass der Text von „Vibrate“ autobiographische Züge trägt. Der Sänger rekonstruiert nach eigener Aus- 
kunft seine - offenbar vergebliche - Suche nach einem Go-Go-Tänzer, mit dem er einmal eine Nacht verbracht hat. ${ }^{5}$ Er streift durch Clubs und Bars, in denen „Electroclash“ läuft, ein zur Zeit der Entstehung des Songs, also um das Jahr 2003, populärer Musikstil in Berlin-Mitte, Brooklyn und anderswo. Im Electroclash wurden neueste elektronische Beats und Sounds mit zu diesem Zeitpunkt bereits als exotisch-primitiv rezipierten Elementen von Post-Punk-Songs der frühen 1980er Jahre oder Electro, einem Seitenarm des frühen HipHop, zu einem referenzreichen, körperlich aktivierenden und, wenn es nach den Promotern und Fans dieser Stilrichtung geht, sexuell stimulierenden Mix verbunden. Und tatsächlich brachte Electroclash in den Clubs ein gegenüber den eingeschliffenen Routinen von House-Music-DJ-Sets neuartiges Kollektiverlebnis hervor, allerdings auch eines mit deutlichen Anzeichen einer Rückkehr zu traditionellen, heterosexuellen Geschlechterrollen. ${ }^{6}$

Das lyrische Ich in Wainwrights Song argwöhnt, die ElectroclashParty sei nichts anderes als „Karaoke“, eine Zuschreibung, die in diesem Kontext kaum aufwertend gemeint sein dürfte. Vielmehr scheint sie als Hinweis auf eine warenförmige-vorgestanzte, ent-individualisierte Performanz zu dienen. Ähnlich vergeblich wie bei der Musik und den körpersprachlichen Angeboten von Britney Spears, deren Name an dieser Stelle nicht nur eine in Musikvideos verbreitete tänzerische Formel, sondern auch so etwas wie die Gesamtheit unauthentischer Pop-Rollenvorbilder für die Jüngeren repräsentiert, ohne dass damit zwangsläufig einer etwaigen Natürlichkeit naiv das Wort geredet würde, sucht es vielmehr nach einer seinem Alter angemessenen Verhaltensform - „I guess, I'm getting on in years".

Während der Protagonist derart als wandelnder Anachronismus durch die Stadt von Electroclash und Britney Spears zieht, ist sein Mobiltelefon auf den vibrate-Modus geschaltet, in der Handy-Industrie bisweilen silent alert genannt. Die vibrate-Einstellung ist auch deshalb nützlich, weil die Räume und Situationen, durch die sich der Suchende bewegt, von lauten Geräuschen und Gesprächen erfüllt sind, von vibrierenden, klangerzeugenden Körpern, die jedes bloß akustische Signal des

5 Vgl. Adams, Tim: „Crystal Clear“, in: The Observer, 20.02.2005, http:// observer.guardian.co.uk/omm/story/0,13887,1416032,00.html, 31.01.2006; siehe auch den „Track-to-Track Commentary“ auf der Homepage von Rufus Wainwright: www.rufuswainwright.com/discography/commentary. asp?album=wantone, 31.01.2006.

6 Vgl. Holert, Tom: „Star-Schnittstelle. Glamour und elektronische Popkultur", in: club transmediale/Jansen, Meike (Hrsg.): Gendertronics. Der Körper in der elektronischen Musik, Frankfurt a.M. 2005, S. 20-43. 
Mobiltelefons übertönen könnten. ${ }^{7}$ Das Telefon und sein Benutzer passen sich diesen Umweltbedingungen an, wechseln das sensorielle Register. Die Mobiltelefon-Forschung spricht hier von „,context awareness“, wobei man mit der situationssensiblen Ausdifferenzierung der akustischen und taktilen Meldesignale der Geräte die Hoffnung auf effektiveres, aber auch rücksichtsvolleres Kommunikationsverhalten verbindet. ${ }^{8}$

Zudem, so der Protagonist, sei völlig unklar, wie sich ,all these new drugs" auf die Wahrnehmung auswirken würden. Dabei geht es ihm gerade darum, nüchtern, alert, alarmierbar, also wach zu sein, rund um die Uhr (,So call me/Call me the morning, call me in the night/So call me/Call me anytime you like“). Der ersehnte Anruf der nächtlichen Bekanntschaft (offenbar wurden Telefonnummern ausgetauscht) darf nicht überhört, nicht überfühlt werden. Oder, wie Wainwright es im Track-toTrack Commentary auf seiner Homepage formuliert: Er habe gewartet, „for my pants to move“". 9

Nicht das Horn des Postillons, die Fahrradklingel des Briefträgers, das Klappern des Briefkastenlids, das Läuten des Telefons, nicht einmal das ,You've got mail‘ eines E-Mail-Programms, sondern eine nichtakustische, nicht-visuelle Störung einer physiologischen, körpereigenen Frequenz oder ,Stimmung' kündigt die Liebeskommunikation an. Andererseits: Wie die voran genannten, historischen, oft auch literarischen, häufig romantischen Beispiele der Ankündigung von Postempfang ist diese Vibration nicht nur somatisch, sondern auch semiotisch. Es richtet sich hier eine hoffende, in den Künsten vielfach präfigurierte Erwartung darauf, dass die begehrte sympathetische Vibration zweier Menschen durch eine forcierte Vibration eingeleitet würde. Denn das Mobiltelefon

7 Die vibrotaktile und elektrotaktile Technologie der Mobiltelefonhersteller steht im engen Zusammenhang mit den haptischen Signalgebern, die für Hör- oder Sehgeschädigte entwickelt werden; die freiwillige oder situationsgebundene Verschiebung bzw. Einschränkung des sensorischen Spektrums ist sowohl prothetisch zu verstehen als auch destabilisierend in Hinblick auf ein etwaiges ideales, ,volles' Körperschema. Vgl. Summers, Ian: Tactile Aids for the Hearing Impaired, London 1992; Chang, Angela u.a.: "ComTouch: Design of a Vibrotactile Communication Device“, in: DIS2002, London 2002, S. 312-320; „Make Technology Work. Report on Technology Access for Deafblind People“, in: Sense, Washington 2005, http://www.incits.org/SWG-A/documents/swga_048.pdf, 31.01.2006.

8 Vgl. z.B. Khalil, Ashraf/Connelly, Kay: „Context-aware configuration: a Study on Improving Cell Phone Awareness“, 2005, www.cs.indiana.edu/ surg/Publications/ContextAwareConfig.pdf, 31.01.2006.

9 Vgl. die Homepage von Rufus Wainwright: www.rufuswainwright.com/ discography/commentary.asp?album=wantone, 31.01.2006. 
vibriert/schwingt - angetrieben durch einen kleinen Motor, der im Geräteinneren eine Unwucht in Bewegung setzt - bei Anruf in einer anderen Frequenz als der Körper, dessen Inhaber es auf sich aufmerksam machen soll. Nur so - indem es dem Körper seines Besitzers eine Abfolge kleiner, elektronisch generierter Schocks versetzt - würde ihn der Anruf erreichen und damit in seiner begehrenden Leiblichkeit bestätigen. Aber diese Schocks, diese punktuelle Reibung, die auch ein erotischer frisson sind, bleiben reine, unerfüllte Möglichkeit. ${ }^{10}$

Der Protagonist rechnet insgeheim mit dieser Unerfüllbarkeit seines Wunsches, denn sein Telefon ist zwar „on vibrate for you“, aber die von Ferne (womöglich überhaupt nicht) ausgelösten Gefühlswellen verfehlen ihn (,I never ever feel from you“). Es gibt weder ein Gefühl, das die Hose, noch eines, das das Herz bewegt. Andererseits heißt es, „Pinocchio" sei nun bereit, vom Subjekt, vom Jungen (,,boy“), zu dem er herangewachsen ist, wieder zum sexuellen Objekt, zum Spielzeug (,toy“) $\mathrm{zu}$ werden. Der Bereitschaft zur Selbstobjektivierung entspricht also (noch) keine reale Gelegenheit.

So könnte man vom Scheitern einer Kommunikation sprechen, von der schleichenden, an manch andere Desillusionierung und Entfremdungserfahrung gebundenen Enttäuschung einer Vibrationserwartung. Jedoch: Auch wenn die Kontaktaufnahme per Mobiltelefon letztlich nicht stattzufinden scheint, so gewährleistet die ,vibrate“-Einstellung des Handys einen subjektiven Zustand der Kommunikabilität und Erreichbarkeit, ein Zustand, der in mancher Hinsicht als Steigerung jener Einschätzung Marshall McLuhans zu betrachten ist, dass das Telefon eine „participant form“ sei, die nach einem Partner verlange (,with all the intensity of electric polarity“) $)^{11}$, selbst dann, wenn dieser sich nicht meldet.

Überdies ließe sich dieser Zustand mit Fredric Jameson als Selbstüberwachung (,,autosurveillance“) charakterisieren, als „Eindringen von Informationstechnologie in den Körper und die Psyche des Individuums": ,[...] es beinhaltet die Verstreuung von Computern auf einem

10 Die sexuellen Konnotationen der Rede vom vibrate sind offenkundig - und auch die vibrate-Funktion des Mobiltelefons wird zur sexuellen Stimulation zweckentfremdet. Darüber informieren z.B. Biddlecombe, Elizabeth: „Sexy Force-Feedback Phone“, in: Wired News, 25.04.2005, www.wired. com/news/wireless/0,1382,67292,00.html, 31.01.2006, sowie der Wikipedia-Eintrag ,vibrating alert“ (http://en.wikipedia.org/wiki/Vibrating_alert, 31.01.2006), in dem auch Wainwrights Song „Vibrate“ kurze Erwähnung findet.

11 McLuhan, Marshall: Understanding Media. The Extensions of Man, New York $^{2}$ 1964, S. 235. 
allgemeinen Maßstab und eine Art passiver Replikation ihrer Programme durch das Individuum. "12 Aber ist diese Replikation wirklich so passiv, und ist der Vorgang, den Jameson meint, überhaupt als Replikation angemessen bezeichnet? Wird nunmehr gekoppelt und determiniert oder nicht vielmehr permanent rückgekoppelt und interagiert?

Rufus Wainwrights Protagonist ist ja nicht nur das willenlose, informationell/medial an das Mobilfunknetz gekoppelte Subjekt einer geschichtsvergessenen und interesselosen Vernetztheit, sondern ein Wanderer im von potentiellen Liebesbotschaften oder -kommandos erfüllten Äther, die auf gepulsten Mikrowellen eintreffen (wenn sie eintreffen); die physischen, sozialen, akustischen, urbanen Räume, die auf der endlosen Suche nach dem Sexualpartner durchquert werden, sind über das Handy im vibrate-Modus an einen intimen Kommunikationsraum angeschlossen, einen Raum, der freilich in diesem Fall genauso mobil ist wie er virtuell bleibt (beziehungsweise arretiert im Stand-by-Betrieb des, per definitionem, unerfüllbaren Begehrens).

\section{Greifbare und utopische Ziele: Innervation und Revolution}

Wainwright schildert mit poetischen und musikalischen Mitteln, was es heißen kann, Telekommunikationstechnologie, die auf die menschliche Physiologie, in diesem Fall: auf die äußere Epidermis einwirkt und die zunehmend pervasiv organisiert ist, in das psychische, soziale und ästhetische Erfahrungsspektrum eines Individuums zu integrieren. Der Song „Vibrate“ zeichnet einen kognitiven Zustand der gesteigerten Erreichbarkeit und Rezeptivität nach, in dem sich das empfindende Subjekt von einem motorisierten Mobiltelefon assistieren lässt. Zugleich wird die haptische Technologie gewissermaßen verspielt, ihre Funktionalitätsansprüche werden ironisiert und in den Kontext einer Camp-Poetik transferiert. Jener „Zustand höchster Disponibilität“, in den die Erwartung eines Anrufes versetzen kann, wie Manfred Schneider für die Telefonepisoden im

$12,,[\ldots]$ autosurveillance marks the penetration of information technology within the body and the psyche of the individual subject: it implies a diffusion of computers on a generalized scale and a kind of passive replication of their programs by the individual“ (Jameson, Fredric: „Foreword“, in: Attali, Jacques: Noise. The Political Economy of Music, Minneapolis/London 1985, S. xiii). 
Werk Marcel Prousts herausgearbeitet hat, ${ }^{13}$ verheißt nicht unbedingt Gutes, sondern kann zur schmerzlichen Konfrontation mit der todesnahen Zerbrechlichkeit der ersehnten Stimme führen. Das Mobiltelefon im vibrate-Modus hütet die Bedrohungen des hedonistischen Umherschweifens.

Was ist also dran an dieser Verbindung zwischen Rezeptivität und latenter Agitation der Haut, an diesem Zustand von Erregung, Empfangsbereitschaft und Mobilität? Lässt sich hier, aber auch in der Art und Weise, wie der Sänger diese Verfassung ästhetisch transformiert, eine Signatur gegenwärtiger Subjektivität entziffern? Um in diesen Fragen weiterzukommen, könnte die Erörterung des Begriffs der Innervation behilflich sein, genauer gesagt, seine Verwendung in den Schriften Walter Benjamins. Das Innervationskonzept bei Benjamin ist in mancher Hinsicht unerklärt und ungeklärt, mit anderen Worten: offen für allerlei Annahmen und Übernahmen. Es bleibt letztlich unentschieden, ob es sich hier um einen Begriff handelt, der im weiteren oder engeren Sinne dem Register von Ästhetik beziehungsweise Aisthetik zugeordnet werden kann, oder ob es sich doch eher um eine Kategorie der Psychologie, Physiologie und deren Manipulation und Optimierung im Arbeitsprozess handelt. Aber gerade diese Instabilität, die sich zwischen ca. 1928 und 1939 wandelnde Funktion und Frequenz der „Innervation“ in Benjamins Medien- und Kunsttheorie(n), könnte eine Reihe gegenwärtiger Probleme und Antinomien rund um Kontrolle und Ermöglichung im Hinblick auf die multisensorielle Bearbeitung, Aktivierung oder Unterwerfung von Körpern in den Medienkulturen erhellen.

Zur Rekonstruktion des Benjaminschen Innervationsbegriffs hat in jüngster Zeit insbesondere Miriam Bratu Hansen beigetragen. ${ }^{14}$ Ihr besonderes Augenmerk gilt der zweiten und für Benjamin definitiven Fassung, dem von Benjamin als „Urtext“ bezeichneten Typoskript, das unter den Materialien des Max-Horkheimer-Archivs in Frankfurt am Main entdeckt und 1989 erstmals veröffentlicht wurde. Aber Hansen verfolgt die Entwicklung des Begriffs nicht nur in den verschiedenen Fas-

13 Schneider, Manfred: „La vitesse des sons. Proust und das Telefon“, in: Felten, Uta/Roloff, Volker (Hrsg.): Proust und die Medien, München 2005, S. 193-212, hier S. 196. Vgl. auch Thomas, Chantal: „From Proust to the Mobile Phone", in: Log. Observations on Architecture and the Contemporary City, H. 2, 2004, S. 65-72.

14 Vgl. vor allem Hansen, Miriam Bratu: „Benjamin and Cinema: Not a OneWay-Street“, in: Critical Inquiry, H. 25, 1999, S. 306-343; sowie dies.: „Room-for-Play: Benjamin's Gamble with Cinema“, in: October, H. 109, 2004, S. 3-45. 
sungen des Kunstwerkaufsatzes, sondern geht der Innervation, diesem ,,antidote - and counterconcept - to technologically multiplied shock and its anaesthetizing economy ${ }^{\text {‘15 }}$ in Benjamins gesamter Produktion der späten 1920er und 1930er Jahre nach. ${ }^{16}$ Sie ist interessiert an der ,Politik der Innervation“"17 in Benjamins Projekt, Formen und Räume einer ,nicht-destruktiven, mimetischen Inkorporation der Welt ${ }^{\star 18} \mathrm{zu}$ erproben. Innervation wäre demzufolge die neurophysiologische Verschränkung mit der Apparatur, eine physische Prädisposition, die dem Kollektiv im Kino sensomotorische Zugriffe auf die anthropologischen Residuen in der Technik ermöglicht - ähnlich wie das im Spiel versunkene Kind oder der Spieler im Kasino mit vor- oder überzivilisatorischen Schichten kommuniziert.

Die große Bedeutung, die der Begriff der Innervation für Benjamin bis 1936 gehabt hat, dokumentiert eine längere Fußnote in der zweiten Fassung des Kunstwerkaufsatzes. In ihr wird die im Haupttext aufgestellte These, dass die ,gesellschaftlich entscheidende Funktion der heutigen Kunst“ (womit vor allem der Film gemeint war) die „Einübung“ in das „Zusammenspiel“ von „Natur und Menschheit“ sei, ${ }^{19}$ durch eine ausführlichere Darlegung des Innervationskonzepts ergänzt. Hatte Benjamin in der ersten Fassung noch sehr knapp die ,geschichtliche Aufgabe“ des Films darin gesehen, die ,ungeheure technische Apparatur unserer Zeit zum Gegenstande der menschlichen Innervation zu machen“ ${ }^{\text {‘20 }}$, so wird er nun, im Rückgriff auf Überlegungen aus den späten 1920er Jahren, ${ }^{21}$ ge-

15 Hansen, Miriam Bratu: „Benjamin and Cinema: Not a One-Way-Street“, in: Critical Inquiry, H. 25, 1999, S. 317.

16 Etwa im Abschnitt „Antiquitäten“ der Einbahnstraße von 1928, wo Benjamin unter der Zwischenüberschrift „Gebetsmühle“ die enge Verbindung von „Vorstellung“ und „Innervation“ im Yoga betont (vgl. Benjamin, Walter: „Einbahnstraße“, in: Gesammelte Schriften, hrsg. v. Rolf Tiedemann/Hermann Schweppenhäuser, Bd. 4, Frankfurt a.M. 1989, S. 116f.).

17 Hansen (wie Anm. 15), S. 340.

18 Hansen, Miriam Bratu: „Room-for-Play: Benjamin's Gamble with Cinema“, in: October, H. 109, 2004, S. 9.

19 Benjamin (wie Anm. 3), S. 359.

20 Benjamin, Walter: „Das Kunstwerk im Zeitalter seiner technischen Reproduzierbarkeit“ (1. Fassung), in: Gesammelte Schriften, hrsg. v. Rolf Tiedemann/Hermann Schweppenhäuser, Bd. 1, Frankfurt a.M. 1989, S. 445.

21 Gemeint sind Benjamins Ausführungen zur revolutionären Innervation des Kollektivs in der Durchdringung von „Leib und Bildraum“ im Surrealismus-Essay von 1929, vgl. „Der Sürrealismus. Die letzte Momentaufnahme der europäischen Intelligenz“, in: ebd., S. 295-310, hier S. 310: „Erst wenn in ihr sich Leib und Bildraum so tief durchdringen, daß alle revolutionäre Spannung leibliche kollektive Innervation, alle leiblichen Innervationen des 
nauer - insbesondere im Hinblick auf die Rolle der Innervation bei der revolutionären Befreiung, die durch „Anpassung“ der „Verfassung der Menschheit“ an die „,neuen Produktivkräfte“ jener „,zweiten Technik“ des Zusammenspiels von Natur und Menschheit erreicht werden könne. ${ }^{22}$

Es ist das Ziel der Revolutionen, diese Anpassung zu beschleunigen. Revolutionen sind Innervationen des Kollektivs: genauer Innervationsversuche des neuen, geschichtlich erstmaligen Kollektivs, das in der zweiten Technik seine Organe hat. Diese zweite Technik ist ein System, in welchem die Bewältigung der gesellschaftlichen Elementarkräfte die Voraussetzung für das Spiel mit den natürlichen darstellt. Wie nun ein Kind, wenn es greifen lernt, die Hand so gut nach dem Mond ausstreckt, wie nach einem Ball, so faßt die Menschheit in ihren Innervationsversuchen neben den greifbaren solche Ziele ins Auge, welche vorerst utopisch sind. Denn es ist ja nicht nur die zweite Technik, die ihre Forderungen an die Gesellschaft in den Revolutionen anmeldet. Eben weil diese zweite Technik auf die zunehmende Befreiung des Menschen aus der Arbeitsfron überhaupt hinauswill, sieht auf der anderen Seite das Individuum mit einem Mal seinen Spielraum unabsehbar erweitert. In diesem Spielraum weiß es noch nicht Bescheid. Aber es meldet seine Forderungen in ihm an. ${ }^{23}$

Dass die Revolution eine Anpassungsleistung voraussetzt, ist eine der dialektischen Pointen in Benjamins geschichtsphilosophischer wie politischer Theorie der Technik und der Medien. Eine andere liegt darin, dass die „Innervationsversuche“ des Kollektivs, also die Emanzipation von der Apparatur, die gerade durch die mimetische Einübung der Apperzeptionen und Reaktionen in sie gelingen soll, vor allem den „Spielraum“ des oder der Einzelnen erweitert. Fast scheint es, als hätte Benjamin die Personalisierungen von Computertechnologie und Telefonie hier mit Hilfe seiner Theorie des Spiels und der Mimesis als emanzipatorische Utopie bereits entworfen. Überdies steckt in der Theorie der Zerstreuung als Individualisierung, wie sie ,nirgends mehr als im Kino“ die „Reaktionen des Einzelnen“ in Hinblick auf ihre „unmittelbar bevorstehende Massierung “24 anschaut - und zwar sowohl in Hinblick auf die faschistische wie auf die proletarische Innervierung des Kollektivs - die ganze Problematik, wie sie die gegenwärtigen, mehr oder weniger ,revolutionären' Theorien und Praktiken auf dem Spektrum von der multitude (Mi-

Kollektivs revolutionäre Entladung werden, hat die Wirklichkeit so sehr sich selbst übertroffen, wie das kommunistische Manifest es fordert.“

22 Benjamin (wie Anm. 3), S. 360.

23 Ebd., Fußnote 4.

24 Ebd., S. 374. 
chael Hardt/Antonio Negri) bis zu den smart mobs (Howard Rheingold) kennzeichnet.

Doch sollte man aus guten Gründen der allfälligen Versuchung, Benjamin zu ,aktualisieren“, mit Vorsicht begegnen. Die Applikation Benjaminscher Begriffe auf das Zeitalter des ,zweiten Medienumbruchs“ ist ein schwieriges Unterfangen, und so belässt es auch Hansen bei Andeutungen, wie sie diese Aktualität begreift. So spricht sie etwa von der Verschaltung ,physiologischer Impulse“ mit „kybernetischen Strukturen“, 25 die sich auf Benjamins Betrachtungen der innervierten Figur der Mickey Mouse zurückführen ließen; durch die Digitalisierung hätten sich zudem die „Spielräume“ geweitet, in denen Benjamins Beobachtungen zum Verhältnis einer Kunst des schönen Scheins einerseits und einer Ästhetik des Spiels andererseits etwa in der Entwicklung von Computerspielen und einem neuen Kino der Attraktionen kulminierten. ${ }^{26}$

Die konkreten Beispiele, die Hansen nennt - das Hongkong-Kino der 1990er Jahre mit seinem hohen Tempo, seiner eskalierenden Gewalt und seinen Techno-Körpern einerseits, sowie die Durchdringung von Techno-Ästhetik, Marketing, Politik und Militär im letzten Golfkrieg andererseits - scheinen eher die Funktion zu haben, ein Forschungsprogramm, das auf der Medien- und Kunsttheorie Benjamins aufbaut (um diese zu überschreiten), anzukündigen, als ein solches Programm bereits umzusetzen.

Diese historische Grundlage allerdings entfaltet Hansen noch einmal sehr überzeugend und auch überraschend aus den Texten heraus, was vor allem daran liegen mag, dass sie den systematisch-strategischen Ort der Innervation, aber auch den des Spiels, bei Benjamin genauer lokalisiert als bisher geschehen. Und letztlich wirft Hansen die Frage auf, wie die Dialektik und die Interaktionen der Innervation bei Benjamin für eine offene, nicht negativistische Anthropologie der Medien unter den politischen, ökonomischen und technischen Bedingungen der Netzwerkgesellschaften zu operationalisieren wären.

Ein zentraler Punkt dieses Projekts ist das spezifische Ermächtigungspotenzial, das Benjamin in der Innervation erkennt. Die neurophysische beziehungsweise psychologische Reizung/Erregung, die sich letztlich motorisch, in Körperbewegungen und -konvulsionen, im Lachen und Weinen im Kinosaal äußert, unterläuft die Schutzmechanismen der Psyche, welche gegen die Realität im tayloristischen Arbeitsprozess aufgebaut wurden.

25 Hansen (wie Anm. 18), S. 41.

26 Vgl. ebd., S. $44 f$. 
Im Zustand der Innervation - verursacht durch das bewegte und montierte (und, wie man sagen muss: stumme) Filmbild und insbesondere durch die für Benjamin avanciertesten Formen der Körperinszenierung beziehungsweise der Ablösung des Körpers vom Schein in der Massenkultur Hollywoods, den Zeichentrickfilmen von Walt Disney und dem „Gestus“ Charlie Chaplins ${ }^{27}$ - wird etwas von der Fähigkeit zur „Erfahrung“ und ihren reflexiven und temporalen Dimensionen eingeholt. Diese Fähigkeit zur Erfahrung drohte ansonsten im Zuge der Industrialisierung des Alltags durch das „Erlebnis“ abgelöst zu werden, welches Reflexion durch Reflexe und historische Zeitlichkeit durch das Diktat des absoluten Präsens ersetzt.

Dass die visionäre Perspektive einer emanzipiatorischen Funktion der „Innervationsversuche“ der Menschheit schwer aufrecht zu erhalten ist, musste Benjamin in dem berühmten Briefwechsel mit Adorno von 1936 feststellen und sie daraufhin, aber auch vor dem Hintergrund des Faschismus, weitgehend relativieren. Allzu dünn war die Materialgrundlage, auf der er sein Argument zu errichten versuchte. Und sie wurde durch die Entwicklung des Tonfilms, durch die Veränderungen in der Anlage der „neuesten Micky-Maus-Filme“, ihre „Neigung, Bestialität und Gewalttat als Begleiterscheinung des Daseins gemütlich in Kauf zu nehmen“28, oder die zunehmende Sentimentalisierung des Spiels von Charlie Chaplin immer dünner.

Gleichgültig ob Benjamin den Begriff nun den zeitgenössischen Diskursen der Neurophysiologie, Psychologie oder Psychoanalyse entlehnt hat - Hansen erkennt in Benjamins Verständnis von Innervation eine mögliche Antwort des Körpers (individuell wie kollektiv) auf die abstumpfenden beziehungsweise routinisierenden Effekte des „Chockerlebnis[ses]“ (Benjamin) in der tayloristischen Arbeitswelt und im technisierten Alltag in den großen Städten. Innervation wäre so betrachtet das Indiz einer nicht mechanischen, sondern mimetischen Rezeption der äußeren Welt, ,die Umwandlung von mentaler, affektiver Energie in somatische, motorische Formen“ sowie die „Konversion und Rettung abgespaltener psychischer Energie durch motorische Stimulation“; diese mimetischen, auch: spielerischen Engführungen von Psychischem und Somatischem, von Energetik und Motorik resultieren in einem ,,porösen

27 Benjamin, Walter: „Varianten und Varia zur ersten Fassung von ,Das Kunstwerk im Zeitalter seiner technischen Reproduzierbarkeit““, in: Benjamin (wie Anm. 20), S. 1040.

28 Benjamin (wie Anm. 3), S. 377, Fußnote 14. 
Interface zwischen dem Organismus und der Welt“, das „eine gesteigerte Mobilität und Zirkulation der psychischen Energien erlaubt“ ${ }^{29}$

Man erahnt in dieser aus Hansens Benjamin-Lektüre gewonnenen Terminologie der Permeabilität und Interaktivität bereits jene Rede von den Kopplungen, die Medientheoretiker heute gern führen, wenn sie „multisensorische Umweltbindungen des Menschen und der Programme“ durch ,,außeninduzierte Erregungen“" ausfindig machen. ${ }^{30}$ Und es wird entscheidend darauf ankommen, wie sich diese „Umweltbindungen“ erklären lassen - und zu welchem Zweck, mit welchem gesellschaftlichen Ziel. Denn es ist gerade das politisch ,Zielführende“ an Benjamins Schriften seit der Mitte der 1920er Jahre, was eine Rekontextualisierung und Aktualisierung sowohl erschwert als auch interessant macht.

Man kann (und sollte) die gegenwärtige Rede von der medialen Kopplung aber auch ins Verhältnis setzen zu den verschiedenen psychotechnischen und soziotechnischen Projekten des, ersten Medienumbruchs'. Hansen erwähnt Benjamins, über Asja Lacis vermittelte Kenntnisse der avantgardistischen sowjetischen Biomechanik-Diskurse bei Meyerhold oder Kuleshov und seine Rezeption von Produktivismus und Operativismus bei Tretjakov. ${ }^{31} \mathrm{Zu}$ denken wäre zudem an den „Fabrikdichter“ Aleksej Gastev, der um 1920 im Moskauer „Zentralinstitut zur wissenschaftlichen Arbeitsorganisation und Mechanisierung des Menschen" mit der Implementierung von tayloristischen Methoden experimentierte, die unmittelbare Folgen für den menschlichen Wahrnehmungsapparat haben sollten. Gastev stützte sich unter anderem auf die Annahme, dass die „Macht des ,Maschinismus““ ein neues „menschliches Sensorium der elektrischen Nerven, Hirnmaschinen und Kinoaugen“ hervorbringe, einen ,globalen, massenhaften Körper mit kollektiven Bewegungen, kollektiven Gefühlen, kollektiven Zielen“632.

Eine andere Arbeit am menschlichen Sensorium, die auch Benjamin bekannt war, ist in den Filmen und Schriften von Vertov oder der Montagetheorie Eisensteins am Werk. Letztere zielte, wiederum von

29 Vgl. Hansen (wie Anm. 15), S. 317.

30 Faßler, Manfred: „Visualisierung epistemischer Objekte“, Vortrag, Karlsruhe 2005, http://web.uni-frankfurt.de/fb09/kulturanthro/d/inst/mafa_texte/ Bild2.pdf, 31.01.2006.

31 Vgl. Hansen (wie Anm. 18), S. 18.

32 Vgl. Buck-Morss, Susan: Dreamworld and Catastrophe. The Passing of Mass Utopia in East and West, Cambridge MA/London 2000, S. 107. Siehe auch: Hellebust, Rolf: „Aleksei Gastev and the Metallization of the Revolutionary Body“, in: Slavic Review, H. 3, 1997, S. 500-518; ders.: Flesh to Metal: Soviet Literature and the Alchemy of Revolution, Ithaca 2003. 
William James beeinflusst, auf die Produktion von Gefühlszuständen durch die filmische Inszenierung und Montage von Körperbewegungen, welche an das mimetische Vermögen der Zuschauer appellieren. ${ }^{33} \mathrm{Ge}$ rade diese dialektische Verknüpfung von gesteigerter Dissoziation und Dehumanisierung einerseits und gesteigertem mimetischem Vermögen in der unwillkürlichen Aneignung und Verkörperung des kinematographischen Bildes durch den individuellen, aber vor allem kollektiven Körper des Filmpublikums anderererseits charakterisiert Benjamins Unternehmen eines ,,anthropologischen Materialismus“334 der Medien.

Nun sollte sich aber die utopisch-messianische Besetzung der Innervation spätestens im Zuge der Arbeit an den vier Fassungen des Kunstwerkaufsatzes - also etwa zwischen 1936 und 1939 - unter dem Eindruck der faschistischen Instrumentalisierung der Medien und der Affektion der Massen in ihr Gegenteil verkehren. Hatte Benjamin im letzten Abschnitt „Zum Planetarium“ im Einbahnstraße-Buch von 1928 einen neuen, durch „Technik“ reorganisierten „Kontakt“ der „Physis“ mit dem „Kosmos“ vorhergesehen, ${ }^{35}$ mit schwer auszurechnenden Folgen zwar, aber immerhin mit der Möglichkeit, dass es das Proletariat ist, welches diese epochale Rekonfiguration des Naturverhältnisses in die Hände nimmt, ${ }^{36}$ so bricht Benjamin - zumindest auf der Textebene - mit dieser Überzeugung im Übergang von der zweiten Fassung des Kunstwerkaufsatzes zur dritten und vierten.

Und wenn er 1939, in seinen Arbeiten zum Baudelaire-Buch, das Erlebnis des Verkehrs als eine Konfrontation mit innervierenden Energien beschreibt, liegen dem bereits einige Jahre der Bearbeitung und Transformation des Begriffs der Innervation zugrunde.

Durch ihn [den Verkehr in der großen Stadt, TH] sich zu bewegen, bedingt für den einzelnen eine Folge von Chocks und von Kollisionen. An den gefährlichen Kreuzungspunkten durchzucken ihn, gleich Stößen einer Batterie, Innervationen in rascher Folge. Baudelaire spricht von dem Mann, der in die Menge eintaucht wie in ein Reservoir elektrischer Energie. ${ }^{37}$

33 Vgl. Hansen (wie Anm. 15), S. $317 f$.

34 Vgl. Benjamin, Walter: „Der Sürrealismus. Die letzte Momentaufnahme der europäischen Intelligenz", in: Benjamin (wie Anm. 21), S. 309.

35 Vgl. Benjamin, Walter: „Einbahnstraße“, in: Benjamin (wie Anm. 16), S. $147 \mathrm{f}$.

36 Vgl. aber Theodor W. Adornos Äußerungen zum „Proletariat (als dem Kinosubjekt)“, Brief an Benjamin vom 18.03.1936, zit. n. Benjamin (wie Anm. 20), S. 1003.

37 Benjamin (wie Anm. 20), S. 630. 
Der Eintritt in das elektrisch aufgeladene Feld des urbanen Lebens ist riskant. Die Innervationen, generiert im von Passanten, Verkehrsmitteln, Geräuschen, Schaufensterauslagen, Plakaten oder Leuchtschriften konstituierten Bildraum, attackieren die Integrität des Subjekts. Dieses ist den Angriffen zunächst wehrlos ausgeliefert. Mehr noch: In Reaktion auf die zivilisatorisch-technische Reizüberflutung bildet es - mit Pawlow argumentiert - Psychosen aus. Der technische und multisensorische Raum der Stadt ist förmlich nicht auszuhalten.

An diesem Punkt der Überlastung, Überreizung, Überforderung greift Benjamin seinerseits auf Freuds energetisches Modell des Reizschutzes zurück. Nach Freud ist der „Reizschutz“, ein Element der traumatischen Neurose. Die psychische Schock-Abschirmung ist mit einem ,eigenen Energievorrat ausgestattet und muss vor allem bestrebt sein, die besonderen Formen der Energieumsetzung, die in ihm spielen, vor dem gleichmachenden, also zerstörenden Einfluss der übergroßen, draußen arbeitenden Energien zu bewahren ${ }^{\text {‘38. }}$.

In fortführender Aneignung dieses Gedankens einer gegen-energetischen Aufrüstung der Psyche des Individuums (die als „Gegenbesetzung“ zugleich eine Reduktion oder Paralyse anderer psychischer Energien ist) interessiert sich Benjamin für die entstehenden Routinen der „Registrierung“ dieser „übergroßen, draußen arbeitenden Energien“, die als „chocks“ auftreten. Die Technik erzieht und formt den Menschen, ,optimiert‘ ihn, führt gewissermaßen ,Updates“ durch; das Bewusstsein entwickelt sich „,im Interesse des Reizschutzes“, wobei „Erfahrung“ zunehmend zum „Erlebnis“ wird, das heißt: dem Schock wird auf Kosten eines etwaigen Inhalts ,eine exakte Zeitstelle im Bewusstsein“ angewiesen. ${ }^{39}$ „So unterwarf die Technik das menschliche Sensorium einem Training komplexer Art", schreibt Benjamin. Aber dabei sollte es nicht bleiben. Denn

[e]s kam der Tag, da einem neuen und dringlichen Reizbedürfnis der Film entsprach. Im Film kommt die chockförmige Wahrnehmung als formales Prinzip zur Geltung. Was am Fließband den Rhythmus der Produktion bestimmt, liegt beim Film dem der Rezeption zugrunde. ${ }^{40}$

38 Freud, Sigmund: „Jenseits des Lustprinzips“ [1920], in: Studienausgabe, hrsg. v. Alexander Mitscherlich u.a., Bd. 3, Frankfurt a.M. 1994, S. 217-72, hier S. 237; von Benjamin zit. in: „Über einige Motive bei Baudelaire“, in: Benjamin (wie Anm. 20), S. 613.

39 „Über einige Motive bei Baudelaire“, in: Benjamin (wie Anm. 20), S. 615.

40 Ebd., S. 630f. 
Womöglich entscheidend ist das mysteriöse ,was“ am Anfang des dritten Satzes dieses Zitats. Dieses Etwas, auf dem die tayloristische Taktung der Industrieproduktion basiert, bedingt zugleich die Rezeption des Films. Benjamin spricht (mit Marx) von der „Arbeitsbedingung“ der kapitalistischen Ordnung und der Maschine, welche die Körper und Psychen der ArbeiterInnen und KonsumentInnen zurichten. Mit einem Begriff, der in dieser Bedeutung etwa dreißig Jahre später geprägt wurde, könnte man dieses Was auch als „Spektakel“ bezeichnen. Auf dem „methodologische[n] Boden dieser Gesellschaft“, schreibt Guy Debord 1967, weitet sich der Bereich der Produktion auf den der Konsumption aus:

Das Spektakel ist [...] die ständige Gegenwart dieser Rechtfertigung [der Bedingungen und der Ziele des bestehenden Systems. $\mathrm{TH}$, als Beschlagnahme des hauptsächlichen Teils der außerhalb der modernen Produktion erlebten Zeit. ${ }^{41}$

In dieser Raum-Zeit des Spektakels werden die Menschen, um wieder zu Benjamin zurückzukehren, zu zuckenden Automaten, ,ihr Verhalten ist [nur noch, TH] eine Reaktion auf Chocks“. ${ }^{42}$ Die Filme von Eisenstein und Vertov, der Gestus von Chaplin, der die „menschliche Ausdrucksbewegung in eine Folge kleinster Innervationen [zerfällt] “433, haben das „Gesetz der filmischen Bilderfolge zum Gesetze der menschlichen Motorik" erhoben, $^{44}$ sie reagieren rezeptiv auf den Input dieser Taktungen und Repetitionen und damit auf das Was der kapitalistischen Ordnung oder Dynamik - ebenso wie die Zuschauer auf diese Ver- und Bearbeitung der Produktionsverhältnisse reagieren, indem sich ihre motorisch-somatischen sowie psychischen Entgegnungen, das Lachen und das Weinen, der Taktung entziehen.

Man könnte auch sagen: dieser Entzug vollzieht sich in Akten der Gegenbesetzung der „, Gegenbesetzung“. Oder: als Kontrollverlust, der sowohl ermächtigende wie entmächtigende Momente hatte, weil das Kino der 1920er Jahre, mit Siegfried Kracauer und Heide Schlüpmann gesagt, ein „Obdach“ bot, in dem man sich ,isoliert vom gesellschaftlichen Zusammenhang“, aber auch „,von der äußeren, physischen Wirklichkeit“" wieder fand, also im Schutz des Kinos die „Erfahrung von der

41 Debord, Guy: Die Gesellschaft des Spektakels, Berlin 1996, S. 17, hier S. 15.

42 Benjamin (wie Anm. 39), S. 632.

43 Benjamin (wie Anm. 27), S. 1040.

44 Ebd. 
Verlorenheit des Menschen“ in dieser Wirklichkeit machen konnte. ${ }^{45}$ Ein Ort, wo im Wechselspiel von mimetischen Prozessen und solchen der Distanzierung die institutionellen Anordnungen der Kulturindustrie reinterpretiert und rekonfiguriert wurden - zumindest potenziell. Zugleich ist der Ort, wo Kontrolle verloren, auch einer, wo Kontrolle ausgeübt wird. Denn, wie Benjamin in der kanonischen Fassung des Kunstwerkaufsatzes schreibt, hier „kontrolliert“ die „Masse“ den Darsteller. Diese „Kontrolle“ der Leistung des Darstellers in der Interaktion mit der „Apparatur" durch ein Publikum, das sich auf seine Weise mit der Apparatur konfrontiert, wird nicht negativ, sondern als zukünftige, brechtianische Möglichkeit ihrer ,politische[n] Auswertung“ beurteilt - dann (aber nur dann), wenn sich der Film ,aus den Fesseln der kapitalistischen Ausbeutung befreit haben wird“46. Ansonsten bliebe es beim Starkult und dem „fauligen Schimmer“ des Warencharakters der Filmdarsteller.

\section{$3 \quad$ Stand-By im Bildraum?}

Wie können nun die verschiedenen Bewertungen und Einordnungen der Innervation durch Benjamin selbst dazu beitragen, die Anrufung der Individuen als Subjekte einer spezifischen Kultur des Stand-By genauer zu bestimmen? Das heißt: die ununterbrochene, flexible Kommunikationsund Interaktionsbereitschaft zu erfassen, wie sie mittels beziehungsweise inmitten von Medientechnologie (auch dann, wenn diese Aktivierung oder Alarmierung aufgeschoben ist, nur in der Antizipation existiert) als ein Prozess sich darbietet, in dem Handlungen und Verhaltensweisen gemäß medienkulturellen Konventionen formiert werden? Diese Konventionen wiederum wandeln sich ihrerseits permanent, wie man inzwischen (und immer noch mit Benjamin) sagen kann: unter dem Eindruck einer medienanthropologisch zu erklärenden Veränderung des „,menschlichen Apperzeptionsapparats “ ${ }^{47}$, der fortwährenden Rekonfiguration des menschlichen Sensoriums, des Zusammenhangs von Wahrnehmungsänderungen und Medienentwicklungen.

Diese Formierungen können mit Benjamin als mehrdeutige und multifunktionale erklärt werden, die sowohl kontrollieren wie ermöglichen, integrieren wie desintegrieren. Keineswegs handelt es sich hier, wie Benjamin bisweilen missverstanden wurde, um die undialektische

45 Schlüpmann, Heide: Öffentliche Intimität. Die Theorie im Kino, Basel/ Frankfurt a.M. 2002, S. 112.

46 Benjamin (wie Anm. 3), S. $369 f$.

47 Benjamin (wie Anm. 27), S. 1049. 
Feier einer Auflösung der Mensch-Maschine-Membran oder um die Rückgewinnung eines menschlichen Instinkts unter Bedingungen der Massenkultur. Aber auch die Annahme einer grundsätzlichen Ambivalenz zwischen Momenten brechtianischer Distanzierung und solchen der Entgrenzung ins Kollektiv und in die Apparatur hinein beseitigt nicht das (keineswegs neue) Problem, wo diese Ambivalenzen zwischen ästhetischer Reflexion, philosophischer Erkenntnis, politischem Handeln und passivem Konsum eigentlich operieren.

Darüber hinaus liegt die Brisanz gerade von Benjamins frühem Verständnis der Innervation (man könnte von ,Innervation I' sprechen) darin, dass er in ihr eine der elementaren Voraussetzungen der surrealistischen, wenn nicht der kommunistischen Revolution ausmacht. Denn das Ineinanderfallen von „Leibraum“ und „Bildraum“, in der sich die „Welt allseitiger und integraler Aktualität" zeige ${ }^{48}$ und die Benjamin im Surrealismus-Aufsatz von 1929 mehr beschwört als beschreibt, ist ohne die „revolutionäre Spannung leibliche[r] kollektive[r] Innervation“ und ohne die „revolutionäre Entladung“ durch die „leiblichen Innervationen des Kollektivs“ nicht zu denken. ${ }^{49}$ Erst diese umfassende (oder besser: massenhafte) Affektion, Erregung und Reizung bewirkt die Überbietung der Wirklichkeit im Sinne des kommunistischen Manifests. Die Innervation vermittelt zwischen der „Physis“ des Kollektivs und einem „hundertprozentigen“, profan erleuchteten „Bildraum“ der Massenkultur - einem Raum, in dem sich Bildlichkeit in radikal neuer Weise organisiert, nämlich als nicht länger „kontemplativ ${ }^{650}$ zu ermessen oder im Hinblick auf diesen Rezeptionsmodus zu produzieren. Kurz darauf, gewissermaßen im Stadium von ,Innervation II‘, wurde für Benjamin aber deutlich, dass die Verabschiedung der Kontemplation Hand in Hand mit der Diagnose der Passivität der Zerstreuung gehen muss. Der Bildraum, in dem und durch den das leibhaftige Kollektiv die Innervation erfährt, soll zum Interaktionsraum werden. Doch wie stellt man sicher, dass aus der entgrenzenden Innervation nicht die durch die „Chockwirkung“ induzierte „gesteigerte Geistesgegenwart ${ }^{\text {} 51}$ wird, die Benjamin in der ersten Fassung des Kunstwerkaufsatzes noch ambivalent als zu erwartende mentale Deformation der Menschheit konstatiert? Zudem ist die Möglichkeit solcher Interaktion an die Bedingung von Besitz- und Kapitalverhältnissen

48 „Der Sürrealismus. Die letzte Momentaufnahme der europäischen Intelligenz", in: Benjamin (wie Anm. 21), S. 309.

49 Ebd., S. 310.

50 Ebd., S. 309.

51 Benjamin (wie Anm. 20), S. 464. 
gebunden. Die Revolution, für die das Massenmedium Film die Ressourcen und den Resonanzraum bereitstellen soll, wird nur dann zur interaktiven Installation, wenn die Verfügungsmacht über die Produktionsmittel an jene „Kämpfer gegen die heutige Gesellschaftsordnung ${ }^{652}$ fällt, mit denen sich Benjamin im Kunstwerkaufsatz zu identifizieren scheint.

Benjamins Vorschläge, im mimetischen Spiel, in der profanen Erleuchtung, im optischen Unbewussten, in der unwillkürlichen Erinnerung und in der Innervation des ineinander fallenden Leib- und Bildraums die manipulativen und depravierenden Effekte der Massenkultur umzulenken und zu unterlaufen, überzeugten weder ihn selbst dauerhaft noch wollen sie etwa siebzig Jahre später unmittelbar einleuchten.

Um zunächst nur einen Punkt herauszugreifen: Allzu undifferenziert erscheint Benjamins Bild der Menge im Kinosaal. Er unterscheidet nicht in Hinblick auf Alter, Geschlecht oder sexuelle Orientierung, auch die Klassenzugehörigkeit der Mitglieder des Publikums bleibt etwas im Dunkeln des Begriffs der „Masse“, den er zwar in die „kompakte, nämlich kleinbürgerliche“ des Faschismus und die „klassenbewusste, nämlich proletarische“ zerlegt ${ }^{53}$, doch taugt das „Proletariat“ des Surrealismus-Essays und späterer Texte kaum als empirische Kategorie (und Benjamin stellte hier den Zusammenhang zum Kinopublikum auch nicht explizit her). ${ }^{54}$ Die Innervation erfasst den einzelnen wie den kollektiven Leib, als würden im „Bildraum“ der später Kulturindustrie genannten Sphäre die sozialen, ethnischen oder sexuellen Differenzen zugunsten eines entdifferenzierten Gesamtkörpers aus lauter Einzelnen aufgehoben.

Zudem spielt die Inszenierung der einzelnen und kollektiven Körper in der Kultur der Weimarer Republik eine kaum zu übersehende Rolle, ja man kann davon sprechen, „dass die Bewohner der zwanziger Jahre des zwanzigsten Jahrhunderts mehr von ihren Körpern besessen waren als irgendeine Generation vor oder nach ihnen in der westlichen Kultur ${ }^{* 55}$.

Vielleicht ließe sich Benjamins Innervation daher auch als (zunächst) ambivalente Faszination für diese Körperlichkeit, für diese Bilder der (nicht nur) filmisch konstruierten und dekonstruierten Körper auch in ihrer neuen Sexualität und Visualität weiterdenken. Dabei gingen

52 Ebd.

53 Benjamin (wie Anm. 3), S. 370, Fußnote 12.

54 Vgl. Hansen (wie Anm. 15), S. 328.

55 Gumbrecht, Hans Ulrich: „Vorwort: Protokoll einer Rettung“, in: Cowan, Michael/Sicks, Kai Marcel (Hrsg.): Leibhaftige Moderne. Körper in Kunst und Massenmedien 1918 bis 1933, Bielefeld 2005, S. 9. 
(und gehen) die Effekte solcher Inszenierungen auf die alltägliche Inszenierung der eigenen Körperlichkeit über die von Benjamin genannten Phänomene hinaus. Die Innervation im Bildraum der Massenmedien erschöpft sich nie in den „Lacheffekte[n]“56 angesichts des Zeichentricks oder der grotesken Slapstick-Einlage, das heißt: dem kollektiven Lachen als „heilsamen Ausbruch derartiger Massenpsychosen“, als „therapeutische Sprengung des Unbewußten“. ${ }^{57}$ Vielmehr greifen die Innervationen im Kino in die Performanzen des Alltags aus. Die mimetischen Prozesse im Zustand der Innervation bleiben nicht auf den physischen Raum des Kinosaals beschränkt, sondern vollziehen sich und setzen sich fort in einer Matrix der Bildräume, die kulturell, historisch und medial mehr oder weniger fest vernetzt sind mit dem des Kinos.

Wie aber sind diese Prozesse und Verhaltensänderungen vermittelt, wie (und für wen) verläuft der Weg von der Innervation zur Interaktion und schließlich zur Revolution - auch wenn eine solche Frage nicht strikt im Sinne Benjamins formuliert sein mag? Sozial, aber auch kognitiv ist die Innervation weder reiner Ordnungs- noch Unordnungszustand. Unklar bleibt freilich, wie und ob Benjamin zwischen kognitiven und sensorischen Prozessen unterscheidet, ob er Innervation überhaupt im Gehirn verortet. Als besonders intensive Involvierung mit und durch die Dingwelt, die im Falle des Kinos zugleich individuell wie kollektiv ist, scheint Benjamin die Spannung zwischen qualitativen und quantitativen Bestimmungen in der Phase von ,Innervation I‘ um jeden Preis aufrecht erhalten zu wollen. Man kann auch fragen, ob bestimmte soziale oder kulturelle Typen, bestimmte „Klassen“ oder ein bestimmtes soziales Geschlecht für Innervation empfänglicher beziehungsweise bereiter sind als andere.

In eine andere Richtung würde die Frage zielen, ob sich die Benjaminsche Innervation der fortwährenden Erzeugung der von Michel Foucault so genannten ,gelehrigen Körper“ verweigert oder öffnet. Jedenfalls ließ sich die Differenz zu Disziplinierung, Gewöhnung und Einübung offenbar nur mühsam und gegen Widerstände behaupten. Selbst dort, wo es um Selbstverlust und Transgression im Rausch ging, bleibt unsicher, inwieweit solche Entregelungen nicht auch nur der Reproduktion von Kreativität dienen und damit kapitalisierbar sind. Andererseits könnte in Benjamins Innervationsbegriff die Intuition wirken, auf die Jo-

56 Kracauer, Siegfried: „Ballett, Jazz, Harold Lloyd“ [1929], in: Werke, hrsg. v. Inka Mülder-Bach, Bd. 6.2.: Kleine Schriften zum Film 1928-1931, Frankfurt a.M. 2004, S. 298.

57 Benjamin (wie Anm. 3), S. 377. 
nathan Crary in Bezug auf die wahrnehmungspsychologischen und neurophysiologischen Konstruktionen eines Subjekts der Aufmerksamkeit seit dem Ende des 19. Jahrhunderts hinweist: Dass nämlich der Fortschritt der Erforschung der Aufmerksamkeit als quantifizierbarer Größe deren eigene „Auslöschung“ enthielt - „,sie erwies sich als kontinuierlich übergehend in Zustände der Zerstreuung, des Wachtraums, der Bewusstseinsspaltung und der Trance“658. Die Ambiguität der Innervation, ihre sowohl mobilisierende wie dezentrierende Tendenz, ist vielleicht gerade Ausdruck einer ebensolchen Indifferenz von Aufmerksamkeit und Zerstreuung. 59

\section{Schock und Berührung}

Um zum Ende doch den Fehler einer ahistorischen ,Anwendung ' zu begehen: Für Benjamin wäre die vibrate-Einstellung eines Mobiltelefons wohl in mancher Hinsicht auch eine Schwundstufe oder auch Wiederentdeckung der Einstellung der „taktilen Rezeption“ oder „taktilen Dominante“, die er als entscheidende Weise der Gewöhnung und der Einübung in Medienveränderungen gegenüber der „optischen Rezeption“ abgegrenzt hat, als tendenzielle Ersetzung des ,gespannten Aufmerkens“ durch das „beiläufige Bemerken“. ${ }^{60}$ Neue Aufgaben der Apperzeption werden, wie Benjamin in der ersten Fassung des Kunstwerkaufsatzes schreibt, in der Zerstreuung ,unter der Hand kontrolliert“. Die ,taktile Dominante“, eigentlich vor allem aus der Architektur bekannt, „regiert“ nun nicht nur die „Umgruppierung der Apperzeption“, sondern auch in die „Optik“ hinein. „Und das eben geschieht im Film durch die Chockwirkung seiner Bilderfolge." Benjamin bestimmt damit zugleich den Film als in seiner Zeit „,wichtigsten Gegenstand“ der „Ästhetik““ 61

Bekanntlich häufen sich in diesen Passagen des Kunstwerkaufsatzes die metaphorischen Anwendungen des Taktilen - von den Kunstwerken der Dadaisten, an denen man „Anstoß“ nimmt, die dem Betrachter „zustoßen“, über das Zustoßende der Traumwahrnehmung bis zum

58 Crary, Jonathan: Aufmerksamkeit. Wahrnehmung und moderne Kultur, Frankfurt a.M. 2002, S. 45.

59 Vgl. ebd., S. 48.

60 Benjamin (wie Anm. 20), S. 466.

61 Vgl. ebd. sowie für die zweite Fassung Benjamin (wie Anm. 3), S. 381. 
Wechsel der Schauplätze und Einstellungen im Film, ,welche stoßweise auf den Beschauer eindringen“"62.

Ein Abschnitt in Benjamins Kunstwerkaufsatz, der in der zweiten Fassung des Textes fehlt, besagt, dass der Film die der „betonten Lebensgefahr, in der die Heutigen leben, entsprechende Kunstform“63 ${ }^{\text {“6ei. }}$ Die mediale Verbindung zwischen Gefahr und Taktilität, Schock und Be-

62 Benjamin (wie Anm. 20), S. 464 und Benjamin (wie Anm. 3), S. 379. Verführerisch wäre es, an dieser Stelle einen Exkurs zu Gilles Deleuze und Henri Bergson einzuschalten, über die Vibration des filmischen Bewegungsbildes, das unmittelbar und materiell am Gehirn rührt, überhaupt zur taktilen Dimension in der Filmtheorie von Deleuze, dass Benjamin seine Konzeption von Innervation und Bildraum entfaltet, ohne sich auf Bergson zu beziehen, genauer auf das erste Kapitel aus Materie und Gedächtnis (1896), aber auch etwa auf die Passagen zum Kinematographen in der Schöpferischen Entwicklung (1907). Der Zusammenhang von Benjamin und Bergson in Fragen von Körper und Bild ist bis heute nicht systematisch entwickelt worden. Dabei hätte die Wiederentdeckung Bergsons für die Medientheorie durch Deleuzes Film-Bücher dazu seit längerem Anlass bieten können. Bergsons Theorie eines Körpers, der selbst Bild ist (und damit unlösbar Teil einer nahen und weiteren Bild-Umwelt, die Bergson ,ensemble d'images“ nennt) und der diejenigen Bilder wahrnehmend filtert und organisiert, die ihn selbst in Bewegung setzen, ließe sich mit den Überlegungen zu Leibraum und Bildraum bei Benjamin in mancher Hinsicht korrelieren. So ist der Begriff des Bildes bei Bergson wie bei Benjamin sehr weit geöffnet und bleibt nicht etwa auf das zwei- oder dreidimensionale visuelle Bild begrenzt. Allerdings geht Benjamin zunächst von einer prinzipiellen Unterscheidbarkeit zwischen Leibraum und Bildraum aus, die erst über den individuellen Rausch oder die kollektive Innervation aufgelöst wird, während Bergson die Bildlichkeit des wahrnehmenden Leibs und die Bildlichkeit des universalen Bild-Ensembles von vorneherein als Ineinander begreift. Und was hat es mit jenem entscheidenden Intervall in Bergsons sensomotorischem Schema auf sich, der zwischen dem empfindungserregenden Sinnesimpuls und dessen Umsetzung in einer Bewegung des wahrnehmenden Körpers liegt? Dieses Intervall ist ein Zeitabstand, eine temporale Lücke. Es ist zugleich das „centre d'indétermination“ (Unbestimmtheitszentrum), als das Bergson den Leib oder vielmehr das Gehirn beschreibt. Bergson spricht vom „bureau téléphonique central“, von der telefonischen Schaltzentrale, die die eingehenden Impulse selegiert und distribuiert. Dieses Unbestimmtheitszentrum, dieser zeitliche Intervall, ist mit Erinnerungen bzw. virtuellen Erinnerungsbildern (Deleuze) gefüllt, die reproduziert werden, um Empfindungen, Handlungen, Bewegungen zu aktualisieren. Es wäre interessant zu untersuchen, ob in Benjamins Konzept der Innervation ein solches Intervall, eine solche Reaktionszeit vorgesehen ist oder ob vielmehr die technisch, sozial und ökonomisch vorangetriebene Dezentrierung des Subjekts in der Moderne ein solches „,centre d'indétermination" tendenziell verschiebt bzw. zum Verschwinden bringt, insbesondere dann, wenn der zeitliche Intervall der Logik der Echtzeit weicht.

63 Ebd., S. 464 (1. Fass.). 
rührung leitet über zur Rolle der vibro-taktilen Technologie, insbesondere, was die neuesten Entwicklungen auf dem Gebiet der Konvergenz von Mobiltelefonie und Computerspielen betrifft und das Design interaktiver Anwendungen sehr weit reichend bestimmen wird.

Etwa ein Vierteljahrhundert nach Benjamins Arbeit am Kunstwerkaufsatz, im Dezember 1959, hielt der Psychologe Frank A. Geldard auf einem Symposium zu „Sensory Communication“ in Chicago einen Vortrag, der sich als so etwas wie ein Ur-Text der heutigen Forschungen zur Vibro-Taktilität erweisen sollte. ${ }^{64}$ Geldard sprach über „Some Neglected Possibilities of Communication" und widmete sich insbesondere der „Haut als Informanten“, weil es sich hier um einen guten „,break-in“ sense" handele. ${ }^{65}$ Kutane Empfindungen, besonders wenn sie durch ungewöhnliche Muster erregt würden, forderten im hohen Maße Aufmerksamkeit. Es sei deshalb möglich und nahe liegend, so Geldard, dass die einfachsten und direktesten Botschaften - nämlich (und hier könnte man eine Verbindung zu Benjamins Engführung von Gefahr und Taktilität sehen) Notfallwarnungen und Alarmmeldungen - über die Haut vermittelt würden. In Geldards Labor an der University of Virginia, wo im Auftrag der US Navy geforscht wurde, widmeten sich die Wissenschaftler der Vermessung und Kartierung der Haut des menschlichen Körpers, um herauszufinden, welche und wie viele kutan vermittelte Impulse gesetzt werden können, um die Grammatik oder Sprache einer „kutanen Kommunikation“ zu entwickeln. Man stellte rasch fest, dass von allen Stimulationen der äußeren Epidermis, und dazu gehören theoretisch chemische, thermale oder elektrische Reizungen, nur die mechanische Vibration die Möglichkeit biete, relativ schmerzfrei und dauerhaft

64 Geldard hatte bereits seit den 1930er Jahren, in Nachfolge der physiologischen Forschungen zum „Vibrationsgefühl“" etwa Max von Freys und im Umfeld von Physiologen wie Louis D. Goodfellow die Wahrnehmung mechanischer Vibration aus psychologischer Sicht erforscht. Vgl. etwa Frank A. Geldard: „Is a Vibratory Sensitivity Mediated by the ,Pressure Sense“?", in: Psychological Bulletin, Bd. 33, 1936, S. 776; ders.: „The Vibratory Response of the Skin and Its Relation to Pressure Sensitivity“, in: Biological Bulletin, Bd. 75, 1938, S. 358-359; sowie Geldards vierteiligen Literaturbericht „The Perception of Mechanical Vibration: I. History of a Controversy“, in: The Journal of General Psychology, Bd. 22, 1940, S. 243-269; „The Perception of Mechanical Vibration: II. The Response of Pressure Receptors“, in: ebd., S. 271-280; „The Perception of Mechanical Vibration: III. The Frequency Function“, in: ebd., S. 281-289 und „The Perception of Mechanical Vibration: IV. Is There a Separate ,Vibratory Sense“?"،, in: ebd., S. 291-308.

65 Vgl. Geldard, Frank A.: „Some Neglected Possibilities of Communication“, in: Science, Bd. 131, Nr. 3413, 27.05.1960, S. 1583-1588. 
über und mit der Haut der Versuchspersonen zu kommunizieren. Ein wichtiges Ergebnis der Experimente bis zum Zeitpunkt des Vortrags und des kurz darauf im Magazin Science publizierten Artikels war die Möglichkeit einer „Sprache der Vibration“. Ähnlich wie das Morsealphabet codiert, setzte sich diese ,,vibratese“ language“ aus unterschiedlichen Einheiten zusammen, die durch Vibrationsfrequenzen, -dauern und -intensitäten unterschieden waren. Ein Versuchssubjekt, das sich dreißig Stunden in dieses Alphabet eingearbeitet hatte, konnte nach weiteren 35 Stunden Übung mit neunzigprozentiger Genauigkeit ganze Sätze über die Haut empfangen, wenn diese in einer Rate von 38 Wörtern mit je fünf Buchstaben pro Minute übertragen wurden. ${ }^{66}$

Die leitende Vorstellung hinter diesen Experimenten war zunächst weniger die Entwicklung einer technischen Kommunikationshilfe für Hör- oder Sehgeschädigte als die Steigerung der sensorisch-semiotischen Effizienz, die umfassende Integration des menschlichen Körpers, vor allem des Jetpiloten, in eine informationelle Infrastruktur, dort, wo eine als gefährlich konstruierte Umwelt ein Höchstmaß an Alarmbereitschaft verlangt.

Die geräuschlose Kommunikation über mechanische Vibration wurde seit den 1950er Jahren systematisch, wenn auch offensichtlich mit Phasen der Stagnation, zu einem den Seh- und Hörsinn ergänzenden Kommunikationskanal ausgebaut. Bedeutende Entwicklungen auf diesem Gebiet brachte die Weltraumforschung mit sich, die etwa der haptisch-robotischen Tele-Chirurgie entscheidende Impulse gab. Die Forschung und Entwicklung von Interfaces im Bereich der Tangible Media, vibro-taktilen Medien oder Haptischen Kollaborationen ist zu einem weiten Praxisfeld angewachsen, wo die wirtschaftliche Auswertung längst begonnen hat. „Now, a mobile that tickles and slaps“, verspricht im Februar 2005 ein Artikel über ein neues Mobiltelefon von Samsung. ${ }^{67}$ „Dieses Samsung handset ist in der Lage so zu vibrieren, dass man der SMS Empfindungen beigeben kann - einschließlich der Möglichkeit, dass sich die Person am anderen Ende so fühlt, als wäre sie geohrfeigt worden." Die zugrunde liegende, von dem Unternehmen Immersion in San Jose entwickelte ,haptic technology“ sei eine komplexere Version der handelsüblichen Handy-Motoren. Frequenz und Amplitude der Vibrationen, die von diesem neuen Modellen generiert würden, simulierten

66 Ebd., S. 1586.

67 Anonym: „Now, a mobile that tickles and slaps“, in: HindustanTimes.comIndo-Asian News Service, 25.02.2005, http://www.hindustantimes.com/ news/181_1256770,00030015.htm, 31.01.2006. 
eine gewünschte Empfindung, in Form von so genannten ,,pre-packaged' haptics“" 68

Dieses sehr buchstäblich Haptische in der Mobil-Tele-Kommunikation und der ihr angeschlossenen Anwendungen wie Computerspiele, das sich hier als eine Ebene der „taktilen Dominante“ etabliert, von der Benjamin in Bezug auf den Film sprach und die Marshall McLuhan dann zur taktil-kinetischen Charakteristik des Raums der elektronischen Medien überhaupt transformieren sollte, scheint mir der Beschäftigung wert - auch und gerade im Rahmen einer Aktualisierung der Benjaminschen Ideen zum Leib- und Bildraum.

Rufus Wainwrights Mitteilung „my phone's on vibrate for you“ signalisiert in diesem Zusammenhang eine durch die Mobiltelefonie (und deren Vorgänger) geprägte und konfigurierte Form der Empfangsbereitschaft, eine historisch und gesellschaftlich spezifische Rezeptivität, deren soziotechnische Voraussetzungen sich von der tayloristischen Physiound Psychomechanik der 1920er Jahre stark unterscheiden, vor deren Hintergrund Benjamin die Innervation als Residuum der „Erfahrung“ gegen den Reizschutz als Funktion des „Erlebnisses“ in Stellung gebracht hat.

Zugleich wäre aber die besondere Innervation zu betonen, die sich in einer solchen medialisierten und zugleich somatisch codierten Empfangs- oder Kommunikationsbereitschaft äußert, wie sie Wainwrights Song natürlich eher ironisch umspielt und disloziert als systematisch entfaltet. Unter anderem regt Wainwright dazu an, die erotische Dimension der Innervation zu entbergen, eine Dimension, die Benjamin nicht unbekannt war und die sich etwa in den kosmologischen Verschmelzungsvisionen einiger Texte der 1920er Jahre recht deutlich äußerte. ${ }^{69}$ Aber noch viel wichtiger scheint, wie Wainwright die Latenz, die aufgeschobene Innervation-qua-Vibration musikalisch und textlich in Szene setzt. Denn er zeigt, dass und wie die Kunst der Popkultur mit den aisthetischen Programmierungen der Technologie ein eigenes und eigensinniges Spiel spielen kann. Die ästhetische Erweiterung des „Spielraums“ distanziert die Anpassung an die Apparatur von sich selbst. Und die Kontrolle und Gestaltung der Innervation durch mobile, vibro-taktile Kommunika-

68 Biever, Celeste: „The Touchy-Feely Side of Telecoms“, in: New Scientist.com, 26.02.2005, www.newscientist.com/article.ns? $\mathrm{id}=\mathrm{dn} 7049,31.01$. 2006.

69 Vgl. hier exemplarisch den Eintrag „Planetarium“ in Einbahnstraße, in: Benjamin (wie Anm. 16), S. 146ff. In seinen Schriften zum Film und zur Kunst vermied es Benjamin jedoch, soweit ich sehe, die Innervation in die Nähe der sexuellen Erregung zu rücken. 
tionsinstrumente, wie sie die Interface-Entwicklung in Aussicht stellt, schafft ja gerade zunehmenden Bedarf für eben solche Distanzierungen in jenen Medienräumen, von denen man immer weniger weiß, wie viel Spielraum sie lassen. 